\title{
The Boundary between the Magnetic Fields of Ap Stars and the Fields of Solar-type Stars
}

\author{
John D. Landstreet \\ Department of Astronomy, The University of Western Ontario \\ London, Ontario N6A 3K7, Canada
}

\begin{abstract}
The boundary between Ap-type magnetic fields and the magnetic fields of solar-type stars occurs near $T_{e} \sim 7000 \mathrm{~K}$, about where deep envelope convection develops in main sequence stars. This seems natural for solar-type stars, in which the field is generated by the convection zone. However, among magnetic Ap stars the frequency of occurrence declines from about $10 \%$ of all $\mathrm{A}$ stars near $\mathrm{A} 0$ to about $1 \%$ near $\mathrm{F} 0$. It is not clear what produces this decline in frequency, but the convection zone is probably not responsible. In fact, it seems likely that if global fossil fields occur in main sequence F stars, such fields should be detectable even if the stars having them are not chemically peculiar.
\end{abstract}

\section{Introduction}

Magnetic fields are observed in two quite different forms in main sequence stars. Among lower main sequence (solar type) stars having $T_{\mathrm{e}} \lessgtr 6000 \mathrm{~K}$, and spectra of G0 or later, some stars have fields which seem to be analogous to the field of the sun. In these stars the fields have rather complex structure and are present in only a part of the photosphere. In contrast, a few percent of upper main sequence stars (Ap or Bp stars), with $7500 \mathrm{~K} \lesssim T_{\mathrm{e}} \lesssim 25,000 \mathrm{~K}$, and spectra between F0 and $B 1$, have quite a different type of field. In these stars, the field is simple in structure, usually roughly dipolar, and appears to cover essentially all of the star.

There is a gap on the main sequence between about F0 and G0 where no magnetic fields are observed. In this paper I shall examine the relevant stellar physics and try to understand why this gap occurs. 


\section{Magnetic field structures on the main sequence}

The differences between the fields of lower and of upper main sequence stars reflect their quite different physical origins. These are summarized in the table below.

\begin{tabular}{|c|c|c|}
\hline & Solar-type stars & Ap- or Bp- type stars \\
\hline $\begin{array}{l}\text { Structure } \\
\text { near surface }\end{array}$ & $\begin{array}{l}\text { Isolated flux tubes } \\
\text { embedded in non-magnetic } \\
\text { regions, as in sun. }\end{array}$ & $\begin{array}{l}\text { Fields permeate entire } \\
\text { atmosphere. Overall } \\
\text { structure usually roughly } \\
\text { dipolar. }\end{array}$ \\
\hline $\begin{array}{l}\text { Strengt of } \\
\text { field }\end{array}$ & $\begin{array}{l}1-5 \mathrm{kG} \text { inside flux tubes, } \\
\text { much less elsewhere. Areal } \\
\text { coverage in various stars } \\
\text { ranges downward from } \sim \\
80 \% \text {; about } 1 \% \text { for sun. }\end{array}$ & $\begin{array}{l}\text { In a single star, field varies } \\
\text { by roughly a factor of } 2 \text { over } \\
\text { surface. In various stars, } \\
\text { mean field ranges from } \\
3 \times 10^{2} \text { to } 3 \times 10^{4} \mathrm{G} \text {. }\end{array}$ \\
\hline $\begin{array}{l}\text { Correlation } \\
\text { with stellar } \\
\text { properties }\end{array}$ & $\begin{array}{l}\text { Areal coverage increases } \\
\text { with decreasing stellar } \\
\text { rotation period. }\end{array}$ & $\begin{array}{l}\text { Field may vary slightly on } \\
\text { average with stellar mass } \\
\text { and/or age. }\end{array}$ \\
\hline $\begin{array}{l}\text { Intrinsic } \\
\text { variability }\end{array}$ & $\begin{array}{l}\text { Pattern of emerging flux } \\
\text { at stellar surface probably } \\
\text { changes on timescale of } \\
\sim 10^{6} \mathrm{~s} \text {. }\end{array}$ & $\begin{array}{l}\text { Field structure seems quite } \\
\text { stable. Observed field varies } \\
\text { only due to stellar rotation. }\end{array}$ \\
\hline $\begin{array}{l}\text { Source of } \\
\text { field }\end{array}$ & $\begin{array}{l}\text { Probably a contemporary } \\
\text { dynamo, either within the } \\
\text { outer convection zone or at } \\
\text { its base. }\end{array}$ & $\begin{array}{l}\text { Probably a fossil field, } \\
\text { compressed from the weak } \\
\text { field of the interstellar } \\
\text { medium as the star } \\
\text { condensed. }\end{array}$ \\
\hline $\begin{array}{l}\text { Evolution of } \\
\text { field }\end{array}$ & $\begin{array}{l}\text { The dynamo is thought to } \\
\text { decline as the rotation of } \\
\text { the star is gradually braked } \\
\text { by its stellar wind. }\end{array}$ & $\begin{array}{l}\text { The field is altered by } \\
\text { changes in stellar structure } \\
\text { as the star evolves, by } \\
\text { meridional circulation, } \\
\text { and by ohmic decay of the } \\
\text { driving currents. }\end{array}$ \\
\hline
\end{tabular}

Observational searches have been made for magnetic fields in the hottest solartype stars by Landstreet (1982) and Gray (1984), among others (see also Borra et al. 1982). Most searches have looked for the coherent fields found in Ap stars, but Gray's survey was sensitive to the disordered fields observed by numerous other observers in cooler stars. No fields have been found in non-Ap stars earlier than G0. Quite probably the absence of fields is related to the dramatic decline of the depth of the surface convection zone with increasing $T_{\mathbf{e}}$. This decline, which is illustrated by Clayton (1983), finds the outer convection zone reduced from a fractional depth of about $30 \%$ of the stellar radius at $T_{\mathrm{e}} \sim 6000 \mathrm{~K}$ to about $5 \%$ at $T_{\mathrm{e}} \sim 7000 \mathrm{~K}$ 
(assuming a mixing length to pressure scale height ratio of $\alpha \sim 1.5$ ). Since it is almost certainly the convection that drives the dynamo which produces fields in solar-type stars, it is reasonable to suppose that as this convection becomes less important with increasing $T_{\mathbf{e}}$, fields should be hard to detect or even non-existent. However, it is not clear just where in this interval the hottest stars with solar-type fields should occur.

Observational searches for fields in upper main sequence stars have shown that fields are always found associated with certain types of atmospheric chemical peculiarity. Fields are found (as a function of increasing $T_{e}$ ) in stars with excess $\mathrm{Sr}, \mathrm{Cr}$, and $\mathrm{Eu}$, with excess $\mathrm{Si}$, with weak $\mathrm{He}$, and at the highest $T_{\mathbf{e}}$ 's, with excess He. These chemically peculiar stars, with their strong magnetic fields, make up about $10 \%$ of all stars with $T_{\mathrm{e}} \sim 12,000-9500 \mathrm{~K}$ (B8-A0), only $\sim 1 \%$ of all late A stars $\left(T_{\mathrm{e}} \sim 8000 \mathrm{~K}\right.$ ), and $0 \%$ of stars later than F0 (Wolff, 1968). The observed fields thus end about where deep convection first begins, somewhere around $T_{\mathrm{e}} \sim$ $7300-7500 \mathrm{~K}$. Is the onset of deep convection related to the low- $T_{\mathrm{e}}$ boundary of the Ap star fields? This is less obvious than the idea that weakening convection leads to an upper boundary for fields of solar-type stars.

\section{Possible explanations of the low $-T_{e}$ boundary of the Ap star fields}

If the deepening convection is to be invoked to explain the cut-off for Ap type magnetic fields around F0, we need to look a little more closely at the interaction between surface convection and a fossil magnetic field.

The hydrostatic and thermal structure of the envelope of a late A type star $\left(T_{\mathrm{e}}=8000 \mathrm{~K}, \log g=4.0\right)$ is illustrated nicely in Figure 1 of Latour, Toomre and Zahn (1981). This model clearly shows the main features expected in stars near the cool boundary of the Ap stars. A surface convection region, caused by partial ionization of $\mathrm{H}$ and $\mathrm{He} \mathrm{I}$, occurs in a region not far below $t \sim 1$, where the pressure $p$ is about $10^{4}$ dynes $\mathrm{cm}^{-2}$. In this region, the thermal energy density in the gas is $(3 / 2) n k T \sim(3 / 2) p$. As long as any convection is subsonic, the kinetic energy in bulk motions should be less than the thermal energy. Thus the total kinetic energy in the gas is of order $2 p$. If the magnetic field has an energy density $B^{2} / 8 \pi$ much greater than $2 p$, it will not be possible for the gas, which is an excellent electrical conductor, to convect, since it would have to strongly distort the field. The largest field in which convection may take place is the equipartition field, given by $B_{\text {eq }}^{2} / 8 \pi \sim 2 p$. For the HI convection zone, $B_{\text {eq }} \sim 10^{3} \mathrm{G}$. In most Ap stars, the surface field is at least this large, and so we expect that this outer convection zone will usually be suppressed by the field. A deeper convection zone, due to partial ionization of $\mathrm{He}$ II (and probably linked to the upper zone by overshooting) occurs at about $10-15,000 \mathrm{~km}$ below the first. Here $B_{\text {eq }} \sim 10^{4} \mathrm{G}$, and in all Ap stars except the very small fraction with the largest fields, convection can persist. However, in slow rotators $\left(v_{\text {eq }} \lesssim 1 \times 10^{2} \mathrm{kms}^{-1}\right)$, which almost all Ap's are, Michaud (1982) has shown that downward diffusion of $\mathrm{He}$ below this second 
convection zone gradually reduces the He concentration to a value too small to drive further convection. Enough He diffusion to shut down this convection zone is expected to occur in about $10^{6} \mathrm{yr}$. Thus, in most magnetic Ap stars, which have main sequence lifetimes of $2 \times 10^{7}-1 \times 10^{9} \mathrm{yrs}$, no convection will occur in the outer envelope.

As one looks at lower values of $T_{e}$, the rapidly deepening zone of convective instability quickly outstrips the ability of any observed magnetic Ap star to control it. Furthermore, because the deepening of the convection zone with lower $T_{\mathrm{e}}$ is due mainly to the low ionization and high opacity of $\mathrm{HI}$ deeper in the envelope, this convection zone cannot be much reduced by He diffusion even if this occurs. Thus, once we reach the values of $T_{\mathrm{e}}$ for which the surface convection zone starts to deepen, convection should occur regardless of whether the star is a magnetic Ap or a normal star.

Now, what sort of effect might a deep convection zone have on a deep-seated fossil field? If we look only at the short interval in $T_{e}$ in which the thickness of the convection zone, from where it is suppressed by the magnetic field to the lower boundary, is no larger than the depth of the stable level above the convection zone (which means as long as the convective zone is no more than a few thousand $\mathrm{km}$ thick), the effect of the convection on the observable field on the stellar atmosphere is not likely to be large. Convective eddies will be smaller than the distance to the photosphere, and although they may locally make bubbles in the otherwise uniform magnetic field lines, the field will have room enough above the convection zone to spread back to a roughly uniform surface flux distribution.

However, as soon as we go to low enough $T_{\mathrm{e}}$ that the thickness of the convective zone substantially exceeds the distance between the photosphere and its upper boundary, the size of the convective eddies should become so large that great distortion of the surface field will occur. Two possible effects seem plausible. One possibility is that the large eddies will confine the surface field to ropes or sheets, with more or less the same total flux emerging from the atmosphere that would occur in the absence of convection, but in a different structure, with larger local fields and a smaller areal coverage. Alternatively, the convection may stir the field lines threading it so vigorously that flux ropes of opposite polarity are pushed together, and reconnection occurs. One would expect that the outer loops would quickly escape from the star, and that the flux lines reconnected deep in the convection zone might stay below it. In this case, substantially less flux might appear in the photosphere than without convection. Only more detailed modelling, or perhaps appropriate observations, can choose among these (or other) possibilities.

However, this possible explanation for the cutoff of Ap star fields at low $T_{e}$ does not account for the decline by a factor of order 10 in the frequency of magnetic stars, as a fraction of all main sequence stars of the same temperature, between A0 and F0. Perhaps deep convection is not the complete explanation of the observed cutoff.

An alternative possibility is suggested by the fact that the Henry Draper spectral type of one of the very coolest magnetic Ap stars, HD 24712 (= HR 1217), is given as F0, rather than F0p. HD 24712 has quite strong (and variable) abundance 
anomalies, including considerable overabundances of several rare earth elements, and a very large magnetic field, of order $5 \mathrm{kG}$ at the surface. It is also near $m_{\mathrm{v}}=6$, so the Harvard objective prism plate should have had an adequate exposure for this star. Nevertheless it was missed by as keen a judge of spectral type as Miss Cannon. This suggests to me that perhaps a substantial fraction of the coolest magnetic chemically peculiar stars may not have been noticed in spectral classification, because the peculiarities are not obvious at low resolution. Since most searches for magnetic fields have concentrated on spectrally classified Ap stars, a substantial selection effect could work against finding Ap-type magnetic fields near the low $-T_{\mathbf{e}}$ boundary.

It thus seems well worthwhile to explore the magnetic fields of the A5 - G0 region again, both from a theoretical and from an observational perspective. There may well be something interesting still to be learned here.

\section{References}

Borra, E.F., Landstreet, J. D., Mestel, L.: 1982, Ann. Rev. Astr. Ap. 20, 191, Table 1. Clayton, D.D.: 1983, Principles of Stellar Evolution and Nucleosynthesis, (Chicago: Univ. of Chicago Press), Sec. 6-6

Gray, D.F.: 1984, Astrophys. J. 277, 640

Landstreet, J.D.: 1982, Astrophys.J. 258, 639

Latour, J., Toomre, J., Zahn, J.-P.: 1981, Astrophys.J. 248, 1081

Michaud, G.F.: 1982, Astrophys.J. 258, 349

Wolff, S.C.: 1968, Pub. Astr. Soc. Pac. 80, 281 